\title{
Circulating Antibody-Secreting Cell Response During Mycoplasma pneumoniae Childhood Pneumonia
}

\author{
Patrick M. Meyer Sauteur, ${ }^{1, \odot}$ Johannes Trück, ${ }^{1,2, \odot}$ Annemarie M. C. van Rossum, ${ }^{3, \bullet}$ and Christoph Berger ${ }^{1, \odot}$
}

${ }^{1}$ Division of Infectious Diseases and Hospital Epidemiology, University Children's Hospital Zurich, Zurich, Switzerland, ${ }^{2}$ Division of Immunology, University Children's Hospital Zurich, Zurich, Switzerland, ${ }^{3}$ Department of Pediatrics, Division of Pediatric Infectious Diseases and Immunology, Erasmus MC University Medical Center-Sophia Children's Hospital, Rotterdam, The Netherlands

Background. We recently demonstrated that the measurement of Mycoplasma pneumoniae (Mp)-specific immunoglobulin (Ig) $\mathrm{M}$ antibody-secreting cells (ASCs) improved diagnosis of $M p$ infection. Here, we aimed to describe $M p$ ASC kinetics and duration in comparison to conventional measures such as pharyngeal $M p$ deoxyribonucleic acid (DNA) and serum antibodies.

Methods. This is a prospective longitudinal study of 63 community-acquired pneumonia (CAP) patients and 21 healthy controls (HCs), 3-18 years of age, from 2016 to 2017. Mycoplasma pneumoniae ASCs measured by enzyme-linked immunospot assay were assessed alongside $M p$ DNA and antibodies during 6-month follow-up.

Results. Mycoplasma pneumoniae ASCs of the isotype IgM were found in 29 (46\%), IgG were found in 27 (43\%), and IgA were found in 27 (43\%) CAP patients. Mycoplasma pneumoniae ASCs were detected from 2 days to a maximum of 6 weeks after symptom onset, whereas Mp DNA and antibodies persisted until 4 months $(P=.03)$ and 6 months $(P<.01)$. Mycoplasma pneumoniae ASCs were undetectable in HCs, in contrast to detection of $M p$ DNA in $10(48 \%)$ or antibodies in $6(29 \%)$ controls for a prolonged time. The Mp ASC response correlated with clinical disease, but it did not differ between patients treated with or without antibiotics against $M p$.

Conclusions. Mycoplasma pneumoniae-specific ASCs are short-lived and associated with clinical disease, making it an optimal resource for determining $M p$ pneumonia etiology.

Keywords. antibiotic; B cell; carriage; diagnosis; vaccination.

Mycoplasma pneumoniae $(\mathrm{MP})$ is a common bacterial pathogen of community-acquired pneumonia (CAP) in children [1]. The "gold standard" for diagnosing $M p$ infection is a $\geq 4$-fold increase in antibody levels [2] but has low sensitivity [3] and is not helpful in acute clinical management because it requires acute and convalescent sera [4]. Single immunoglobulin (Ig) M levels against $M p$ and $M p$-specific polymerase chain reaction (PCR) on upper respiratory tract (URT) samples are currently used to diagnose $M p$ CAP $[5,6]$. However, these diagnostic tests are also positive in asymptomatic carriers, and therefore they are unable to differentiate between $M p$ infection and carriage [7]. We recently demonstrated that the measurement of $M p$ specific IgM antibody-secreting cells (ASCs) by enzyme-linked immunospot (ELISpot) assay improved diagnosis of $M p$ infection: $M p$-specific IgM ASCs were detectable in children with $M p$ CAP but not in $M p$ carriers suffering from CAP caused by other pathogens or asymptomatic $M p$ carriers [8].

Received 3 December 2019; editorial decision 29 January 2020; accepted 6 February 2020; published online February 8, 2020.

Correspondence: Patrick M. Meyer Sauteur, MD, PhD, Division of Infectious Diseases and Hospital Epidemiology, University Children's Hospital Zurich, Steinwiesstrasse 75, CH-8032 Zurich, Switzerland (patrick.meyer@kispi.uzh.ch).

The Journal of Infectious Diseases ${ }^{\circledR} \quad$ 2020;222:136-47

(C) The Author(s) 2020. Published by Oxford University Press for the Infectious Diseases Society of America. All rights reserved. For permissions, e-mail: journals.permissions@oup.com. DOI: 10.1093/infdis/jiaa062
Previous work in vaccine studies has established that circulating antigen-specific B-cell responses are more rapid and shorter lived than antibody responses $[9,10]$. Antigen-specific $B$ cells proliferate and differentiate after antigen exposure into ASCs and memory B cells $[11,12]$. Antibody-secreting cells then circulate in the peripheral blood for up to 2 weeks before (1) migrating to secondary lymphoid organs or bone marrow or (2) undergoing apoptosis $[12,13]$. However, data on the human B-cell response during infection rather than following controlled antigen challenge via vaccination are scarce. In a longitudinal follow-up of our recent study [8], we aimed to describe the onset, kinetics, duration, and isotype of the antigen-specific plasmablast response after $M p$ infection in children in comparison to more conventional measures such as $M p$ deoxyribonucleic acid (DNA) in the URT and serum antibody responses. We further evaluated the effect of different treatment regimens against $M p$ on the ASC response and these other measures.

\section{METHODS}

\section{Ethics Statement}

The ethics committee of Zurich, Switzerland, approved the protocol for this study (no. 2016-00148). Written informed consent was obtained from all parents and from children from 14 years of age. 


\section{Participants}

We consecutively enrolled patients between May 1, 2016 and April 30, 2017, at University Children's Hospital Zurich. In all children, pharyngeal swab specimens were taken. Blood samples were collected if additional consent was given. All participants were invited to participate in this longitudinal follow-up study with additional visits between enrollment-2 weeks, 2 weeks -2 months, and/or 2-6 months after presentation.

\section{Community-Acquired Pneumonia Patients}

Community-acquired pneumonia was defined as a clinical diagnosis of pneumonia in previously healthy children aged 3-18 years, as detailed elsewhere [8, 14]. Trained physicians identified cases at the emergency department and on pediatric wards. Community-acquired pneumonia patients $<3$ years of age were excluded to reduce the probability of viral infection, because it is highest in this age group [1, 15-17]. Communityacquired pneumonia patients were managed by treating physicians, who were not aware of the study test results, according to current guidelines $[5,6]$.

\section{Healthy Controls}

Healthy controls (HCs) included healthy children undergoing elective surgical procedures and siblings of CAP patients. Healthy children undergoing elective surgical procedures were age-matched and excluded if there was a history of a recent $(\leq 1$ week) respiratory tract infection. Samples were collected at enrollment by the attending anesthesiologist before surgery while the child was under general anesthesia. We also recruited and followed siblings of CAP patients without evidence of recent respiratory tract infection.

\section{Study Procedures}

\section{Collection of Biological Samples}

Swabs were taken from the posterior pharynx using flocked nylon fiber tip swabs (Copan Diagnostics, Murrieta, CA). Blood samples were collected in anticoagulated lithium heparin blood collection tubes, and fresh peripheral blood mononuclear cells (PBMCs) were isolated $\leq 4$ hours after sampling to avoid poor assay performance due to decreased ASC viability. The PBMCs were isolated by density gradient centrifugation with Ficoll-Paque PLUS (GE Healthcare, Uppsala, Sweden), and viability was assessed by trypan blue exclusion. Serum was stored at $-80^{\circ} \mathrm{C}$.

\section{Mycoplasma pneumoniae Real-Time Polymerase Chain Reaction}

Deoxyribonucleic acid isolation was performed on pharyngeal swab samples with the QIAamp DNA Mini Kit (QIAGEN, Hilden, Germany). A quantitative TaqMan (Applied Biosystems, Foster City, CA) real-time PCR assay was used to detect and quantify $M p$ DNA as described previously [18].

\section{Mycoplasma pneumoniae-Specific Enzyme-Linked}

\section{Immunosorbent Assay}

Mycoplasma pneumoniae-specific IgM, IgG, and IgA antibody levels were determined using a commercially available enzymelinked immunosorbent assay (Virion\Serion, Würzburg, Germany), according to the manufacturer's instructions.

\section{Mycoplasma pneumoniae-Specific Antibody-Secreting Cell Enzyme-}

\section{Linked Immunospot Assay}

The frequency of circulating $M p$-specific IgM, IgG, and IgA ASCs was measured by ELISpot assay using fresh PBMCs, as described previously [19], with some modifications. In brief, 96-well ELISpot filter plates (Millipore, Billerica, MA) were coated for 90 minutes at $37^{\circ} \mathrm{C}$ with the different antigens diluted in sterile phosphate-buffered saline (PBS). The antigens were as follows: detergent extract of $M p$ enriched for highly specific adhesion protein P1 (2 $\mu \mathrm{g} / \mathrm{mL}$; Virion $\backslash$ Serion); Fluarix Tetra quadrivalent influenza $A$ and $B$ virus vaccine (6 $\mu \mathrm{g} / \mathrm{mL}$; GlaxoSmithKline, Middlesex, UK); and affinitypurified antibodies to human Ig light chains ( $\lambda$ and $\kappa, 10 \mu \mathrm{g} /$ $\mathrm{mL}$; SouthernBiotech, Birmingham, AL) as the positive control. The negative control consisted of PBS only in uncoated wells. After washing, coated plates were blocked with medium for another 90 minutes at $37^{\circ} \mathrm{C}$. Coated plates were incubated at $37^{\circ} \mathrm{C}$ for $16-20$ hours with 100000 or 10000 viable PBMCs, and each dilution was used in triplicate. Plates were then washed, incubated with biotinylated anti-IgM, -IgG, -IgA and alkaline phosphatase (AP)-conjugated streptavidin (all SouthernBiotech), and spots visualized using an AP substrate kit (Bio-Rad Laboratories, Hercules, CA), with each spot appearing at the former location of a single ASC. Spots were counted by an ELISpot reader (AID, Strassberg, Germany) using predefined settings. The spots identified by the machine were manually inspected for the presence of artifacts. Antigen-specific spot counts were calculated as the mean of 3 wells minus the mean number of spots in PBS wells. Data were expressed as ASCs per $10^{6}$ viable PBMCs [19].

\section{Statistical Analysis}

We report dichotomous variables as percentages and continuous variables as medians with interquartile ranges (IQRs). The Mann-Whitney $U$ test was used to compare medians, and the Fisher exact test was used to compare proportions. Spearman rank correlation was used to evaluate relationships between variables. Kinetics were plotted with smooth curves, fitted by loess (with a span of 0.67) using the scatter. smooth formula [20]. Study design and sample size considerations were described in detail previously [8]. All reported $P$ values are 2 -tailed with statistical significance defined as $P$ $<.05$. Data were analyzed using the $\mathrm{R}$ software environment (version 3.6.0) [20]. 


\section{RESULTS}

\section{Study Population}

During the 12-month study period, we screened 450 CAP patients and $156 \mathrm{HCs}$ (Figure 1). A total of 152 CAP patients and 156 HCs were enrolled (Table 1). Mycoplasma pneumoniaespecific PCR and serological test results of the enrolled population are shown in Table 2. We included 63 CAP patients and 21 HCs with fresh (isolated $\leq 4$ hours) PBMCs available for additional testing with the $M p$-specific ASC ELISpot assay, as previously reported [8]. There were no statistically significant differences between included and overall enrolled CAP patients and HCs in regards to age, sex, and season at enrollment (Table 1). However, the proportion of siblings of index patients among HCs was higher in the included group of controls compared with enrolled controls ( $43 \%$ vs $12 \%, P<.01)$. Clinical, radiological, and laboratory characteristics of included CAP patients have been described separately $[14,21]$.

\section{Kinetics and Duration of the Antibody-Secreting Cell Response}

We first assessed the ASC response at presentation and found $M p$-specific ASCs in CAP patients $(n=63)$ of the isotype IgM, IgG, and IgA in 29 (46\%), 27 (43\%), and 27 (43\%), respectively (Table 2). These first samples were collected at a median of 12 days after onset of symptoms (IQR, 11-16; range, 2-29). Then, the median number of Mp IgM ASCs was 690 (IQR, 200-1933) spots per $10^{6}$ PBMCs, which was higher than that of $M p$ IgG ASCs (median 300; IQR, 166-533; $P=.07$ ) and $M p$ IgA ASCs (median 167; IQR, 67-333; $P<.01$ ). Mycoplasma pneumoniaespecific IgM ASCs correlated with Mp IgG ASCs (rho 0.63; $P<.01$ ) and $M p$ IgA ASCs (rho $0.62 ; P<.01$ ) but not with $M p$ antibody (any isotype) or $M p$ DNA levels (Supplementary Table 1). Time since onset of symptoms positively correlated with $M p$ IgM and $M p$ IgG antibody levels (rho 0.56 and $0.53 ; P<.01$ ), but not with $M p$ ASCs (any isotype), in first measured sample (Supplementary Table 2). Among HCs $(n=21)$, no Mp ASCs were detected, but $M p$ IgM serology was positive in 6 (29\%) individuals, 1 (5\%) of whom showed seroconversion to $M p$ IgG with a $>4$-fold increase in $M p$ IgG (Table 2). All $29 M p$ IgM ASC-positive CAP patients were also $M p$ PCR-positive and $M p$ IgM-positive, and the 3 (5\%) Mp PCR-positive patients, who were $M p$ IgM ASC- and $M p$ IgM-negative, were identified as $M p$ carriers suffering from CAP caused by other pathogens, as detailed elsewhere [8]. The specificity of the $M p$ IgM ASC ELISpot assay was further demonstrated by the absence of $M p$ IgM ASCs in influenza virus [14] and Epstein-Barr virus (EBV) [22] infected patients (Supplementary Figure 1). The 2 patients who tested positive for influenza virus in pharyngeal swab samples [14] had influenza-specific but not $M p$-specific ASCs detectable during CAP.

The longitudinal follow-up study included 52 (62\%) children (41 CAP patients and $11 \mathrm{HCs}$ ) with $>2$ visits in $42(81 \%)$ and $>3$ visits in 27 (52\%) children, performed at $<2$ weeks, 2 weeks-2 months, and 2-6 months in 43 (83\%), 38 (73\%), and 38 (73\%) individuals, respectively. Mycoplasma pneumoniae
A

CAP

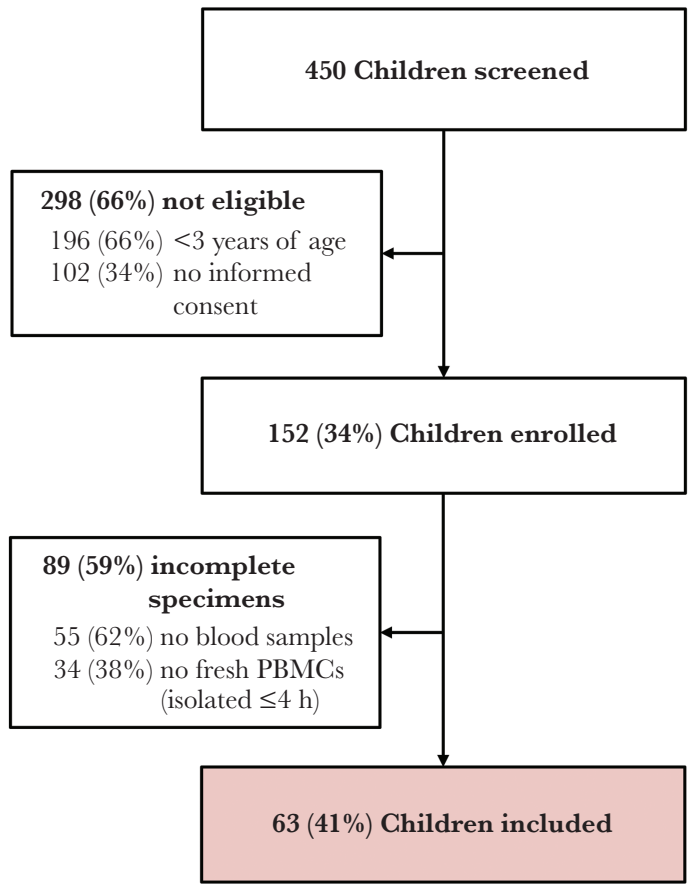

B

$\mathrm{HC}$

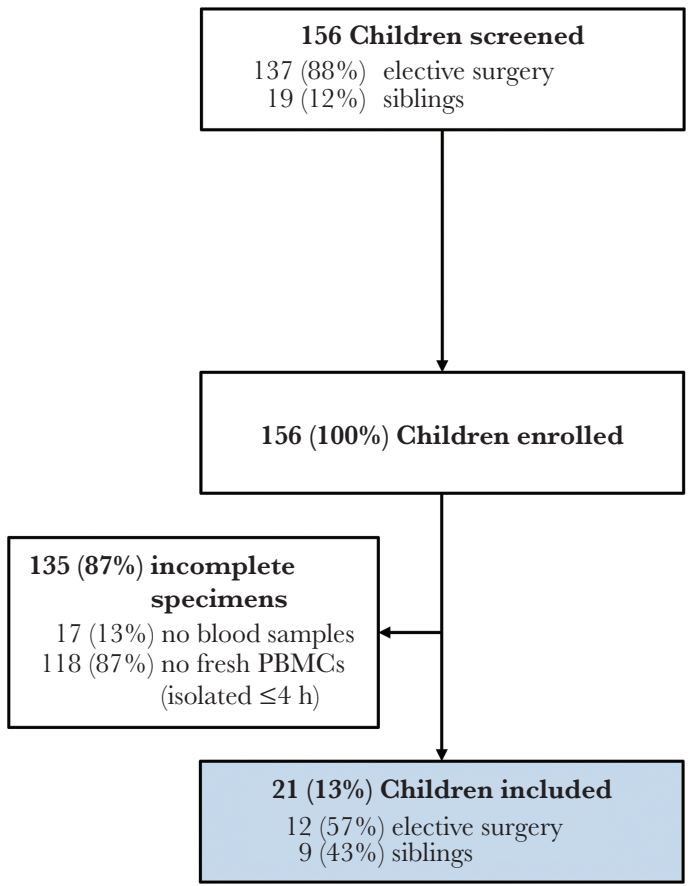

Figure 1. Study profile. Recruitment and flow of (A) community-acquired pneumonia (CAP) patients and (B) healthy controls (HC). PBMC, peripheral blood mononuclear cells. 


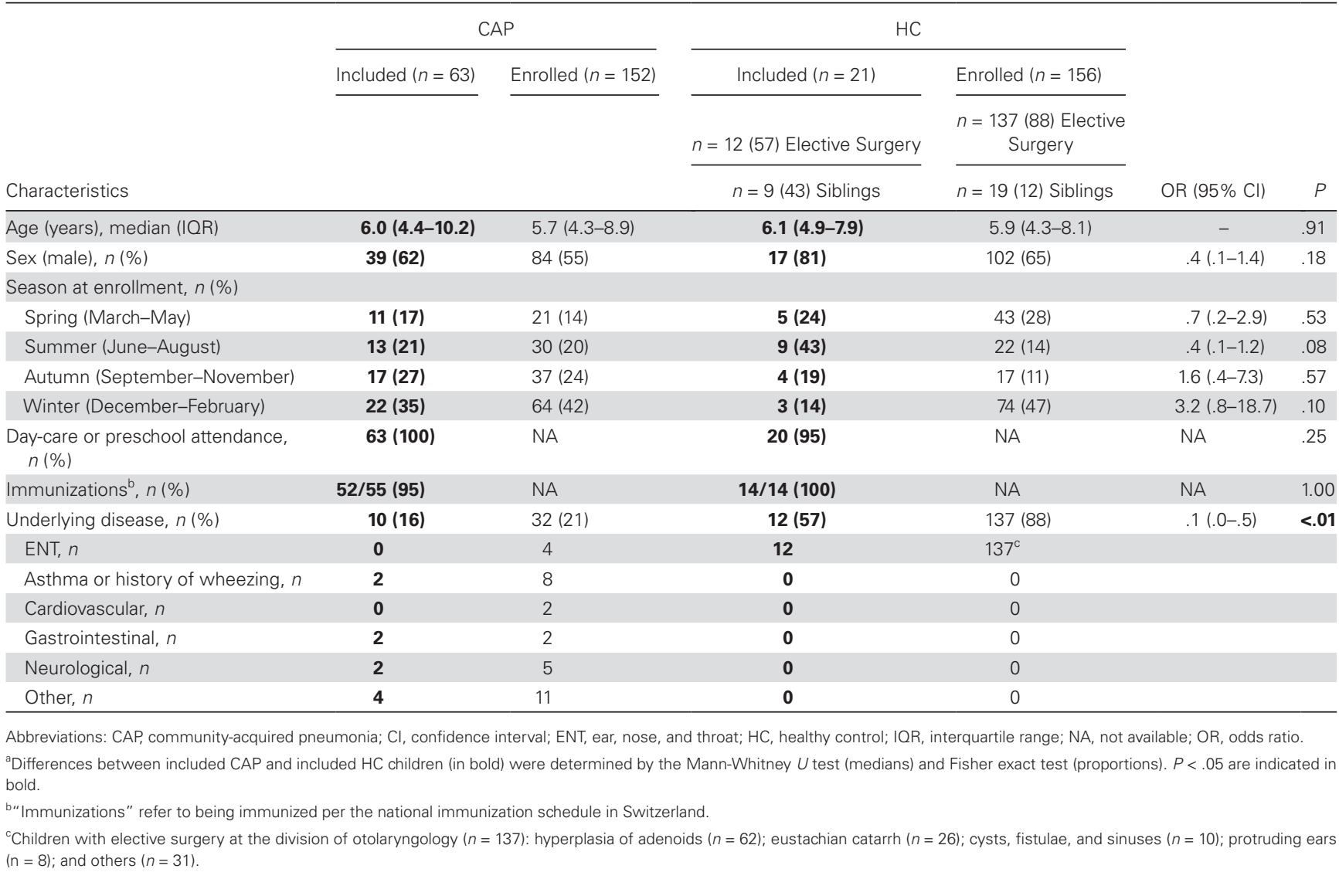

ASCs were detected only within 6 weeks after symptom onset (Figure 2), in contrast to $M p$ DNA $(P=.03)$ and $M p \operatorname{IgM}$ and $M p \operatorname{IgG}(P<.01)$ persisting at 4 and 6 months, respectively (Supplementary Table 3 ). The $M p$ IgA levels in CAP patients were low (median $11 \mathrm{U} / \mathrm{mL}$, IQR 6-25) and above the cutoff in only $13(21 \%)$ (Table 2 ) and differed significantly from $M p \operatorname{IgM}(P<.01)$ and $M p \operatorname{IgG}(P<.01)$ levels. However, $M p$ antibody levels and kinetics correlated between isotypes (Supplementary Table 1 and Figure 2). Detecting a $\geq 4$-fold $M p$ IgG increase in $M p$ IgM ASC-positive patients was significantly more likely with $M p \operatorname{IgG}<20 \mathrm{U} / \mathrm{mL}(91 \%, n=10$ of 11) compared with $M p \operatorname{IgG} \geq 20 \mathrm{U} / \mathrm{mL}$ ( $0 \%, n=0$ of 18 ; $P<.01)$ at presentation.

It is interesting to note that 3 siblings of CAP patients developed CAP during follow-up and were then also tested positive for $M p$ IgM ASCs. One of the 3 siblings was sampled (as asymptomatic sibling of a CAP patient) 3 days before the onset of symptoms, and at this time point, the sibling tested negative by the $M p$ ASC ELISpot assay, followed by a positive $M p$ ASC ELISpot assay result 6 days after developing symptoms and CAP diagnosis. Representative $M p$ IgM ASC ELISpot assay responses in this patient are shown in Figure 3. The kinetics of $M p$ DNA, $M p$ antibodies, and $M p$ ASCs of this patient are indicated in black in Supplementary Figure 2.
In HCs, $M p$ DNA and/or $M p$ IgM and IgG were detected for up to $\geq 2$ months, whereas $M p$ ASCs and $M p$ IgA levels were negative during the complete 6-month follow-up period (Figure 4). These results were in line with the lack of respiratory symptoms during follow-up visits.

\section{Relation of the Antibody-Secreting Cell Response With Clinical Disease}

We next compared the ASC response with clinical disease. As previously demonstrated in this cohort [14], Mp IgM ASC-positive CAP was statistically associated with prolonged prodromal symptoms and extrapulmonary manifestations, predominantly skin disorders [21]. However, the magnitude of the $M p$-specific ASC response did not correlate with any of these clinical features (Supplementary Table 2). Furthermore, lower levels of C-reactive protein (CRP), white blood cell (WBC) count, absolute neutrophil count (ANC), and procalcitonin were statistically associated with IgM ASC-positive $M p$ infection [14]. Mycoplasma pneumoniae IgM ASC responses correlated positively with CRP levels (rho $0.45 ; P=.01$ ), and negatively with WBC count (rho $-.61 ; P<.01$ ) and ANC (rho -.66; $P<.01$ ), but not with other laboratory parameters (Supplementary Table 2).

Next, we evaluated the effect of different treatment regimens against $M p$ on the ASC response. Ten (34.5\%) patients received 
Table 2. Mp-Specific Test Results of Enrolled and Included CAP Patients and Controls ${ }^{\mathrm{a}}$

\begin{tabular}{|c|c|c|c|c|}
\hline Diagnostic Test & CAP & $\mathrm{HC}$ & OR $(95 \% \mathrm{Cl})$ & $P$ \\
\hline Enrolled Cohort & $(n=152)$ & $(n=156)$ & & \\
\hline$M p$-specific DNA & $44(29)$ & $\begin{array}{l}12(8) \\
6 / 137 \text { (4) elective surgery } \\
6 / 19 \text { (32) siblings }\end{array}$ & $4.9(2.4-10.6)$ & $<.01$ \\
\hline \multicolumn{5}{|l|}{ Mp-specific Antibodies } \\
\hline $\lg ^{+}$ & $39 / 97^{*}(40)$ & $15 / 139 *(11)$ & $5.5(2.7-11.7)$ & $<.01$ \\
\hline $\operatorname{lgG}^{+}$ & $39 / 97(40)$ & $16 / 139(12)$ & $5.1(2.6-10.7)$ & $<.01$ \\
\hline $\lg \mathrm{A}^{+}$ & 24/97 (25) & $0 / 139(0)$ & NA & $<.01$ \\
\hline $\lg ^{+} \operatorname{lgG}^{+}$ & $37 / 97$ (38) & $7 / 139(5)$ & $11.5(4.7-32.3)$ & $<.01$ \\
\hline $\lg \mathrm{M}^{+} \lg \mathrm{A}^{+}$ & $24 / 97(25)$ & 0/139 (0) & NA & $<.01$ \\
\hline $\lg G^{+} \lg A^{+}$ & 23/97 (24) & $0 / 139(0)$ & NA & $<.01$ \\
\hline $\lg ^{+} \lg G^{+} \lg A^{+}$ & 23/97 (24) & 0/139(0) & NA & $<.01$ \\
\hline Included Cohort & $(n=63)$ & $(n=21)$ & & \\
\hline Mp-specific DNA & $32(51)$ & $\begin{array}{l}10(48) \\
4 / 12(33) \text { elective surgery } \\
6 / 9(66) \text { siblings }\end{array}$ & $1.1(.4-3.5)$ & 1.00 \\
\hline \multicolumn{5}{|l|}{ Mp-specific Antibodies } \\
\hline $\lg ^{+}$ & $32(51)$ & $6(29)$ & $2.6(.8-9.1)$ & .08 \\
\hline $\operatorname{lgG}^{+}$ & $27(43)$ & $5(24)$ & $2.4(.7-9.4)$ & .19 \\
\hline $\lg A^{+}$ & $13(21)$ & $0(0)$ & NA & .03 \\
\hline $\lg ^{+} \operatorname{lgG}^{+}$ & $26(41)$ & $5(24)$ & $2.2(.7-8.8)$ & .20 \\
\hline $\lg ^{+} \lg \mathrm{A}^{+}$ & $13(21)$ & $0(0)$ & NA & .03 \\
\hline $\operatorname{lgG}^{+} \lg A^{+}$ & $11(17)$ & $0(0)$ & NA & .06 \\
\hline $\lg ^{+} \lg G^{+} \lg A^{+}$ & $11(17)$ & $0(0)$ & NA & .06 \\
\hline \multicolumn{5}{|l|}{ Seroconversion: } \\
\hline $\lg M$ & $0 / 38 * *(0)$ & $0 / 11 * *(0)$ & - & 1.00 \\
\hline $\lg G$ & $5 / 38(13)$ & $1 / 11(9)$ & $1.5(.1-78.7)$ & 1.00 \\
\hline $\lg A$ & $5 / 38(13)$ & $0 / 11(0)$ & NA & .57 \\
\hline Class switch from $\lg M$ to $\lg G$ & $7 / 38(18)$ & $1 / 11(9)$ & $2.2(.2-111.7)$ & .66 \\
\hline \multicolumn{5}{|l|}{ Titer Increase: } \\
\hline $\lg M \geq 2$-fold [3] & $3 / 38(8)$ & $0 / 11(0)$ & NA & 1.00 \\
\hline $\operatorname{lgG} \geq 4$-fold [2] & 10/38 (26) & $1 / 11(9)$ & $3.5(.4-170.1)$ & .41 \\
\hline \multicolumn{5}{|l|}{ Mp-specific ASC } \\
\hline $\operatorname{lgM} \mathrm{ASC}^{+}$ & $29(46)$ & $0(0)$ & NA & $<.01$ \\
\hline $\lg \mathrm{ASC}^{+}$ & $27(43)$ & $0(0)$ & NA & $<.01$ \\
\hline $\lg \mathrm{ASC}^{+}$ & $27(43)$ & $0(0)$ & NA & $<.01$ \\
\hline $\operatorname{lgM} \mathrm{ASC}^{+} \operatorname{lgG} \mathrm{ASC}^{+}$ & $27(43)$ & $0(0)$ & NA & $<.01$ \\
\hline $\lg M \mathrm{ASC}^{+} \lg \mathrm{A} \mathrm{ASC}^{+}$ & $27(43)$ & $0(0)$ & NA & $<.01$ \\
\hline $\lg G \mathrm{ASC}^{+} \operatorname{Ig} \mathrm{A} \mathrm{ASC} C^{+}$ & $26(41)$ & $0(0)$ & NA & $<.01$ \\
\hline $\operatorname{lgM~ASC}^{+} \lg G \mathrm{ASC}^{+} \lg \mathrm{A} \mathrm{ASC}^{+}$ & $26(41)$ & $0(0)$ & NA & $<.01$ \\
\hline
\end{tabular}

Abbreviations: ASC, antibody-secreting cells; CAP, community-acquired pneumonia; $\mathrm{Cl}$, confidence interval; DNA, deoxyribonucleic acid; $\mathrm{HC}$, healthy control; Ig, immunoglobulin; Mp, Mycoplasma pneumoniae; NA, not available; OR, odds ratio.

${ }^{a}$ Data are presented as no. (\%). Differences between groups are indicated by the Fisher exact test. $P<.05$ are indicated in bold.

* Sera were available in 97 (64\%) CAP patients and $139(89 \%)$ controls.

** Paired sera were available in $38(60 \%)$ CAP patients and $11(52 \%)$ controls.

clarithromycin, 10 (34.5\%) patients received doxycycline, and 9 (31\%) patients were not treated with any antibiotic in vitro active against $M p$ (ie, amoxicillin [ $n=5]$, amoxicillin-clavulanate $[n=1]$, ceftriaxone $[n=1]$, no antibiotic treatment $[n=2])$. Kinetics of pharyngeal $M p$ DNA, $M p$ antibodies, and $M p$ ASCs according to different treatment management are shown in Figure 5. There were no statistically significant differences between patients with or without treatment in vitro active against $M p$ with regards to ASC and antibody responses (Supplementary
Table 4). In contrast, $M p$ DNA was detected in patients treated with clarithromycin and doxycycline in $58 \%$ and $90 \%$ at 1 - to 2-month follow-up $(P=.01)$ and $38 \%$ and $0 \%$ at 3 - to 4 -month follow-up $(P=.20)$, respectively. It is notable that clinical outcome (length of hospital stay and long-term sequelae) and fever duration was not statistically different between treatment groups (Supplementary Figure 3). Five patients who received antibiotics against $M p$ were additionally treated with corticosteroids because of severe extrapulmonary mucocutaneous 
A

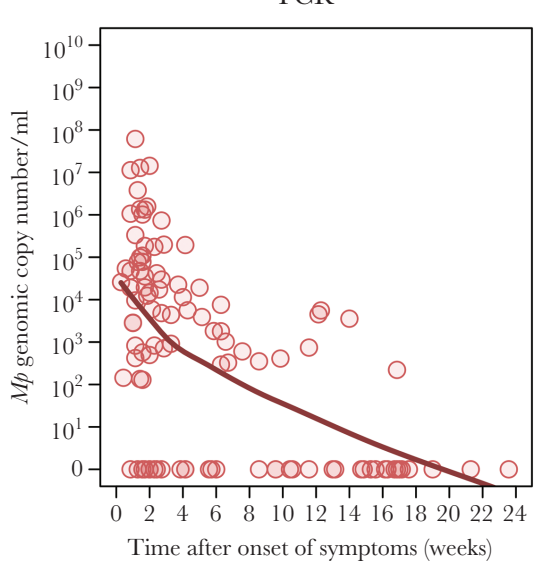

B

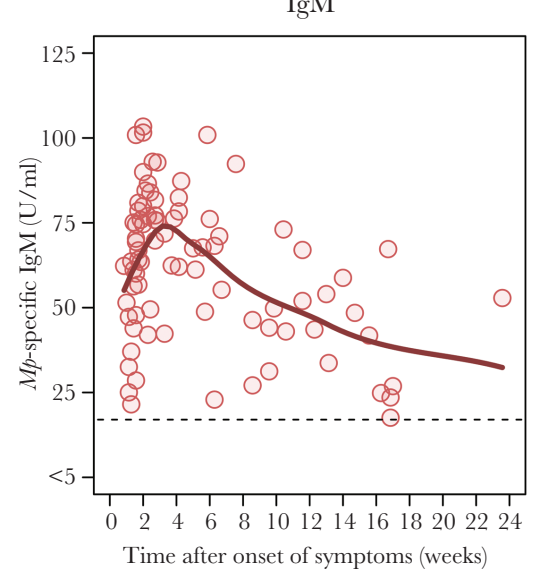

G

IgG

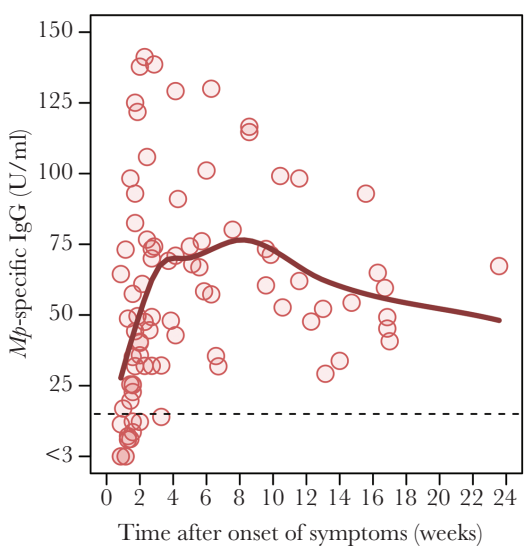

$\mathrm{D}$

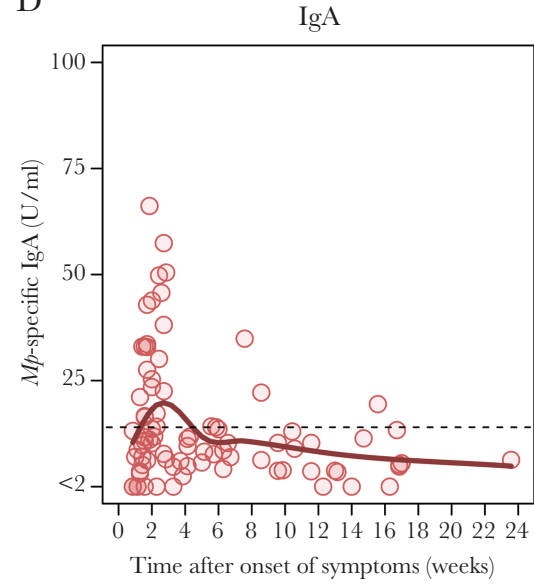

IgM ASC

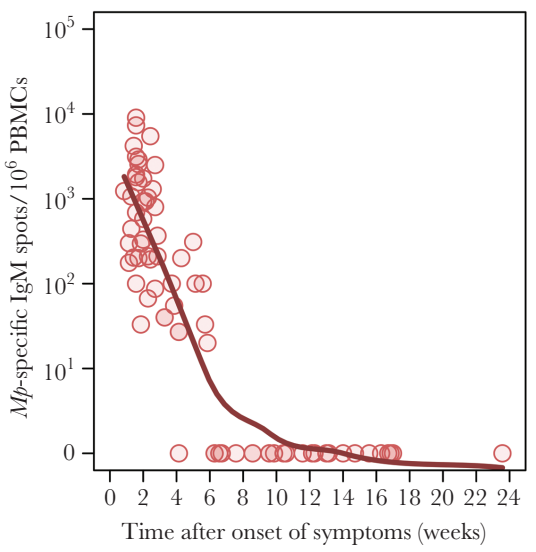

IgG ASC

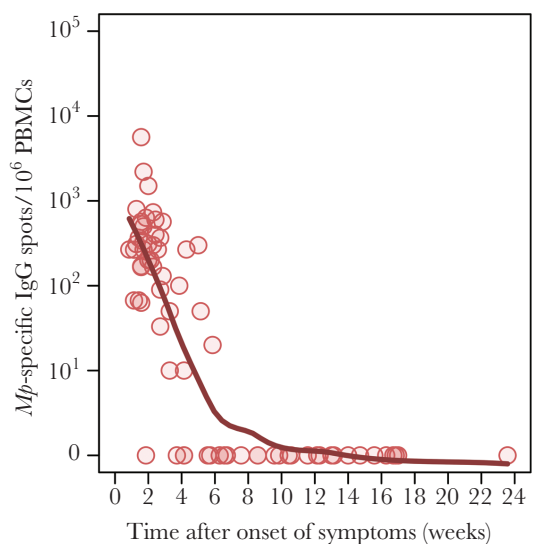

IgA ASC

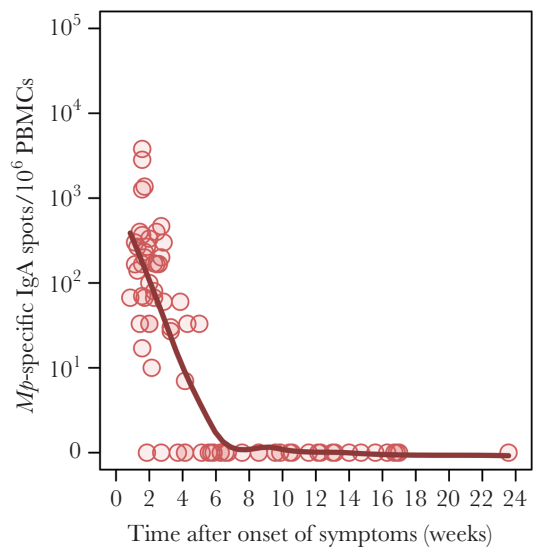

Figure 2. Kinetics of pharyngeal Mycoplasma pneumoniae (Mp) deoxyribonucleic acid (DNA) levels, $M p$ antibodies, and $M p$ antibody-secreting cells (ASCs) in $M p$ immunoglobulin (Ig) M ASC-positive community-acquired pneumonia patients. (A) Mycoplasma pneumoniae DNA levels in pharyngeal swab samples as genomic copy number per milliliter. (B-D) Mycoplasma pneumoniae antibodies and Mp ASCs of the isotype $\operatorname{lgM}(\mathrm{B}), \operatorname{lgG}(\mathrm{C})$, and $\lg \mathrm{A}(\mathrm{D})$. Smooth curves fitted by loess (with a span of 0.67 ) and original data as dots are shown. The dashed horizontal lines represent the cutoff for the enzyme-linked immunosorbent assay, and lower limits of quantification for IgM, IgG, and IgA are 5, 3, and $2 \mathrm{U} / \mathrm{mL}$. PBMC, peripheral blood mononuclear cell; PCR, polymerase chain reaction.

manifestations [21]. Mycoplasma pneumoniae IgM ASC numbers did not differ between those patients before corticosteroid treatment and other CAP patients at an early time point $(<2$ weeks after onset of symptoms) $(P=.49)$, but they were significantly lower at a later time point (2-4 weeks after onset of symptoms) after the administration of corticosteroids compared with 


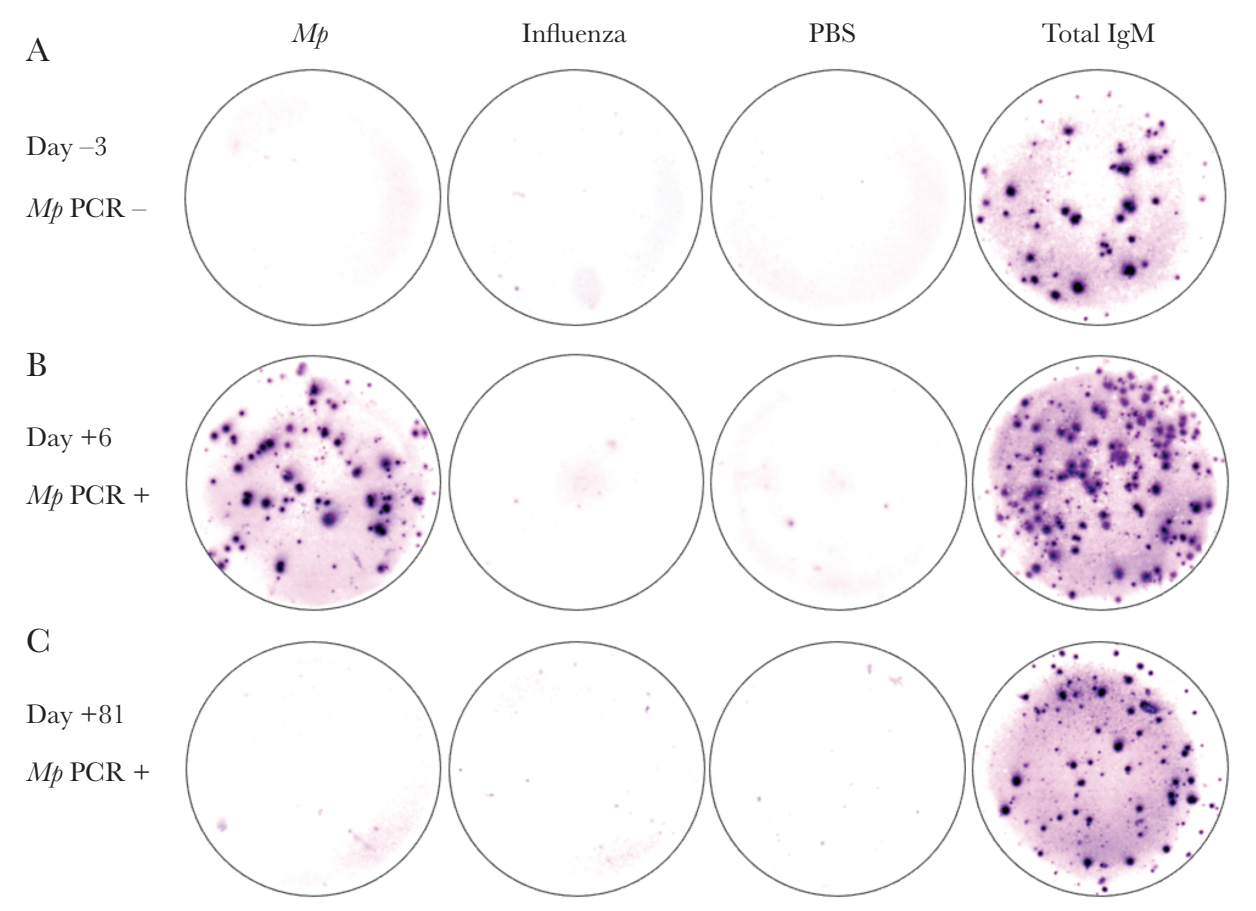

Figure 3. Enzyme-linked immunospot (ELISpot) assay for antibody-secreting cells in a patient who developed Mycoplasma pneumoniae (Mp) community-acquired pneumonia. Representative patterns of ELISpot assay wells 3 days before the onset of symptoms (A) and on day 6 (B) and day 81 (C) after symptom onset (100 000 peripheral blood mononuclear cells per well). Ig, immunoglobulin; PBS, phosphate-buffered saline; PCR, polymerase chain reaction.

CAP patients without corticosteroid treatment sampled at the same time period $(P=.04)$ (Supplementary Figure 4$)$.

\section{DISCUSSION}

In this longitudinal follow-up study, we demonstrate that $M p$ specific ASCs are short-lived and associated with clinical disease, in contrast to pharyngeal $M p$ DNA and serum antibodies that can persist for months and may be present also during $M p$ carriage. This study adds to our recent work [8] by detailing the onset, kinetics, duration, and isotype (IgM/IgG/IgA) of the antigen-specific plasmablast response after $M p$ infection in children and investigating the effect of different treatment regimens against $M p$ on the specific immune response and pharyngeal $M p$ DNA load. These findings expand our current knowledge on specific B-cell responses to infection and provide an explanation for the high specificity and sensitivity of the $M p$ ASC ELISpot assay during $M p$ CAP [8]. The data presented give insight into disease pathophysiology and can therefore serve as a model for developing better diagnostic tests for other childhood infectious diseases.

We detected Mp ASCs as early as 2 days after the presentation of clinical symptoms, which was in line with the only previous study on $M p$-specific ASCs including $12 \mathrm{Mp}$-seropositive children with CAP, in which $M p$ ASCs were detected within 5 days after symptom onset [23]. To our knowledge, we assessed also for the first time B-cell responses in a patient shortly before the development of symptoms and could thereby exemplarily demonstrate a specific ASC response upon CAP onset, which disappeared again with disease resolution, whereas $M p$ DNA and antibodies persisted for prolonged time. Mycoplasma pneumoniae-specific ASCs peaked approximately 1-2 weeks after onset of symptoms, similar to previous work: we recently reviewed the literature on the ASC response to infection [9] and found that the timing of ASC appearance in the blood is highly consistent after infection across several pathogens. Antibody-secreting cells are first detectable in peripheral blood at approximately 4 days after onset of symptoms before peaking at days 6-8 $[9,24]$. In contrast to the conserved timing of ASC appearance, the magnitude of ASC responses varies widely between different bacterial and viral pathogens as well as between ASC isotypes [9]. Our results are consistent with previous work studying the immune responses to both vaccination [13] and infection [9], and they demonstrate a synchronized response of B cells with different isotypes during infection. In contrast, serum antibody responses showed a sequential appearance in our study: IgM and IgA were first detectable followed by $\operatorname{IgG}$ with concentrations that were still rising in the first 4 weeks after symptom onset and lasting for several months.

However, in some individuals, B-cell responses could be detected up to 6 weeks after onset of symptoms, indicating that $M p$-specific ASCs may circulate longer compared with other (respiratory) infections, in which antigen-specific ASCs disappeared in most cases already 14 days after symptom onset $[9,25-30]$. These findings support the clinical observation of 
A

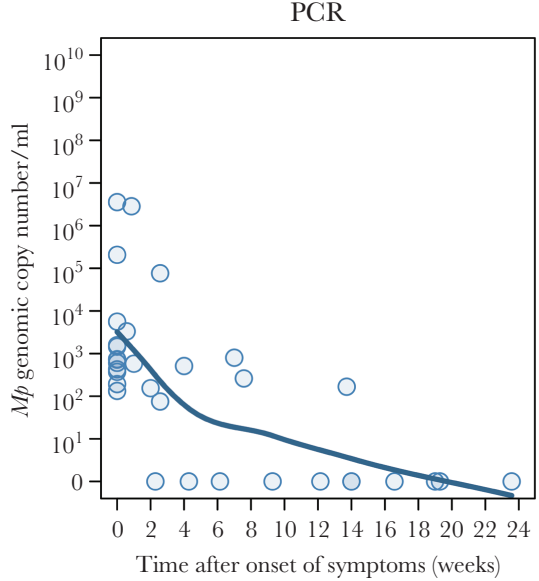

B

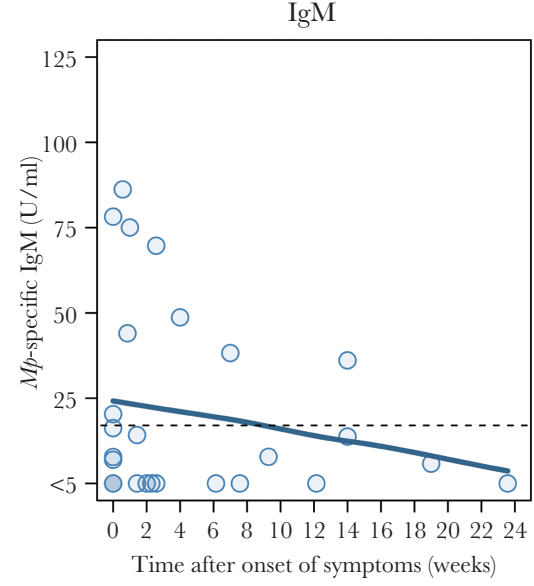

C

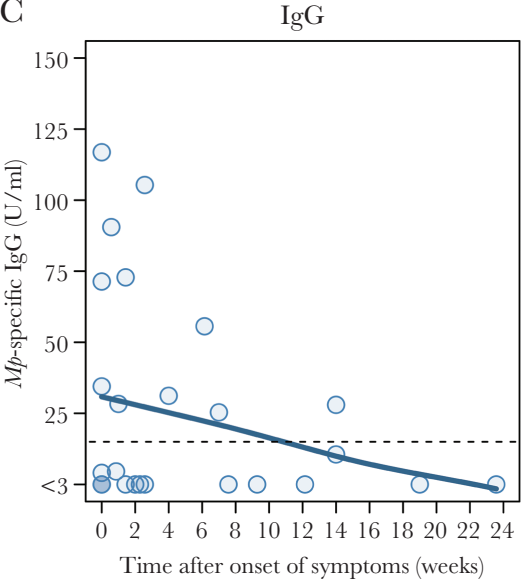

D

IgA

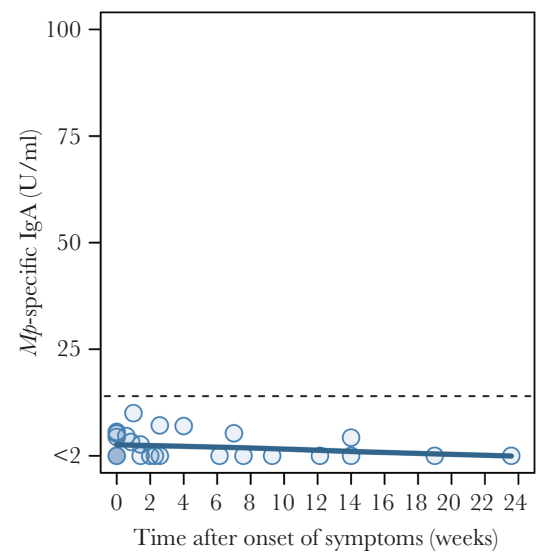

$\operatorname{IgM~ASC~}$

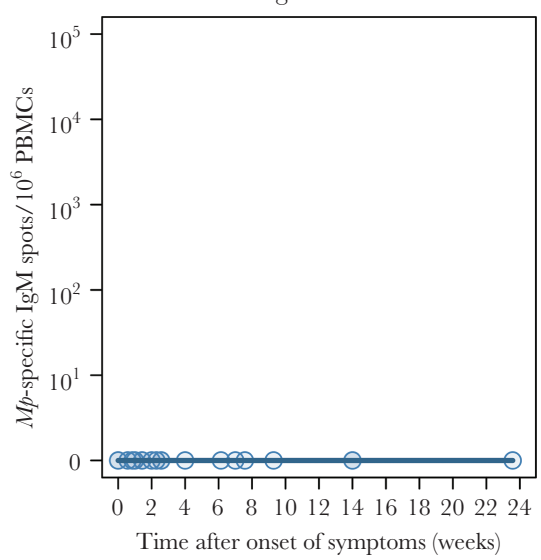

$\operatorname{IgG}$ ASC

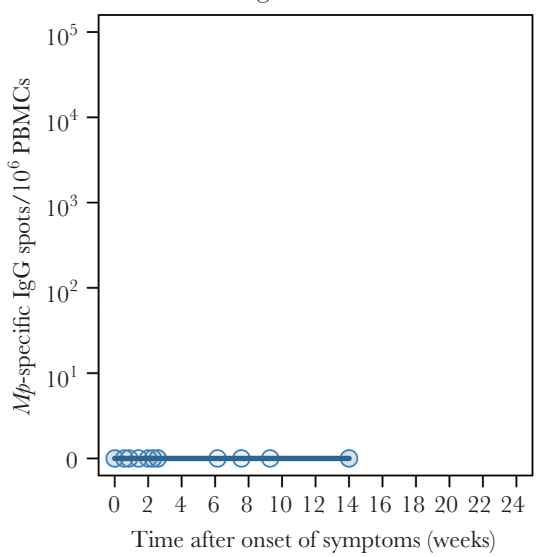

IgA ASC

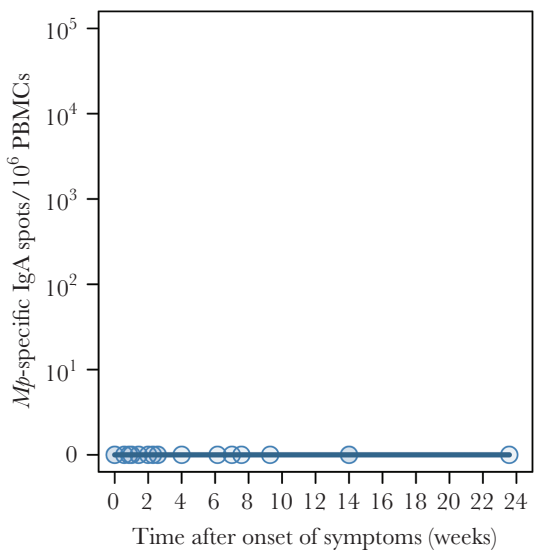

Figure 4. Kinetics of pharyngeal Mycoplasma pneumoniae ( $M p$ ) deoxyribonucleic acid (DNA) levels, $M p$ antibodies, and $M p$ antibody-secreting cells (ASCs) in $M p$ polymerase chain reaction (PCR)-positive controls. (A) Mycoplasma pneumoniae DNA levels in pharyngeal swab samples as genomic copy number per milliliter. (B-D) Mycoplasma pneumoniae antibodies and Mp ASCs of the isotype immunoglobulin (Ig) M (B), IgG (C), and IgA (D). Smooth curves fitted by loess (with a span of 0.67) and original data as dots are shown. The dashed horizontal lines represent the cutoff for the enzyme-linked immunosorbent assay, and lower limits of quantification for IgM, IgG, and IgA are 5 , 3 , and $2 \mathrm{U} / \mathrm{mL}$. PBMC, peripheral blood mononuclear cell.

slow disease progression in Mp CAP [14], which may result in a longer antigen exposure and therefore immune activation, which allows detection of $M p$-specific ASCs in all patients during CAP. A more prolonged antigen exposure with slow disease progression may also be the reason for the significantly increased $M p$ IgG levels already in the first serum sample of $M p$ 
PCR

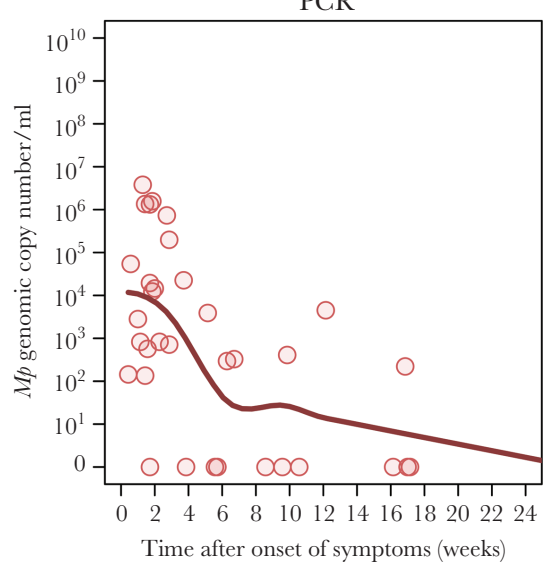

B Macrolide

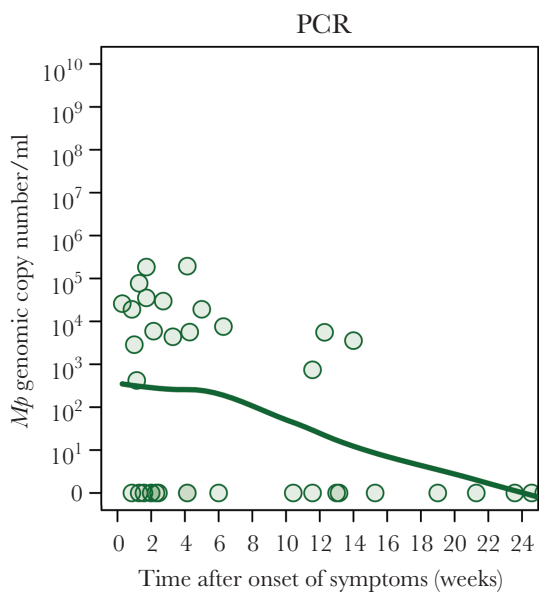

G Doxycycline

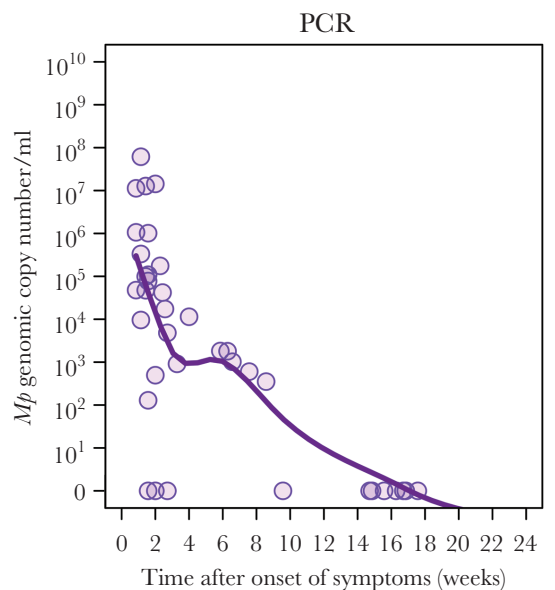

$\operatorname{IgM}$
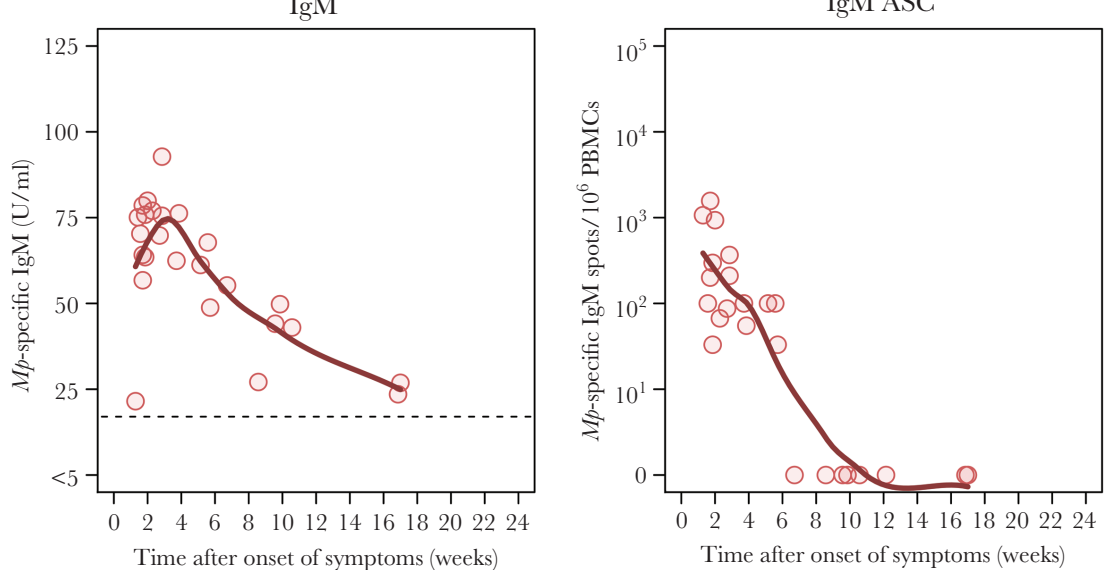

$\operatorname{IgM}$
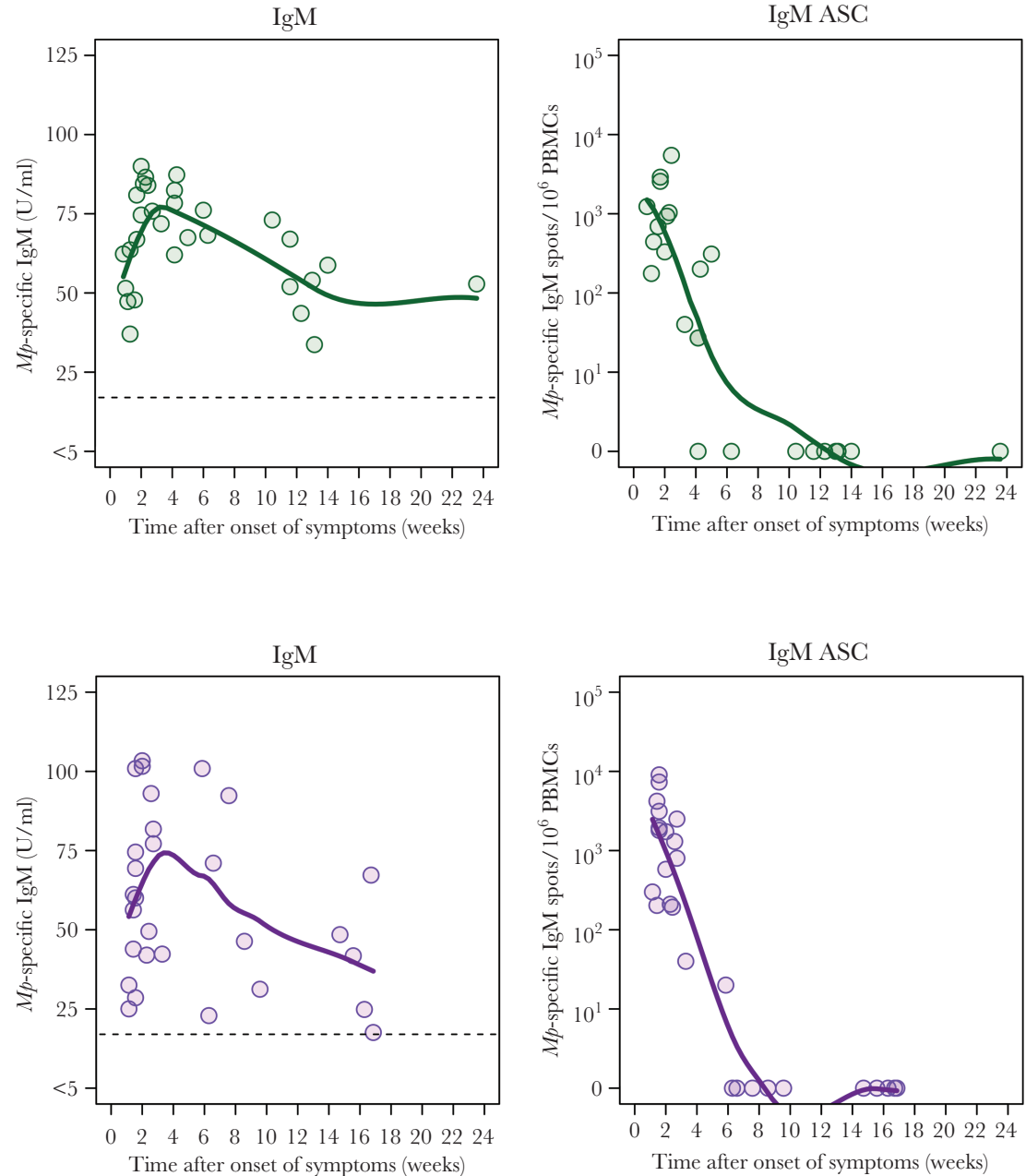

Figure 5. Kinetics of pharyngeal Mycoplasma pneumoniae (Mp) deoxyribonucleic acid levels, Mp antibodies, and $M p$ antibody-secreting cells (ASCs) in $M p$ immunoglobulin (Ig) M ASC-positive community-acquired pneumonia patients according to different treatment management. (A) $\beta$-lactam antibiotics $(n=7)$ or no treatment $(n=2)$. (B) Macrolide (clarithromycin) $(n=10)$. (C) Doxycycline $(n=10)$. Smooth curves fitted by loess (with a span of 0.67$)$ and original data as dots are shown. The dashed horizontal lines represent the cutoff for the enzyme-linked immunosorbent assay (lower limit of quantification, $5 \mathrm{U} / \mathrm{mL}$ ). PBMC, peripheral blood mononuclear cell; PCR, polymerase chain reaction. 
IgM ASC-positive CAP patients. The development of a further increase of $M p$ IgG in those patients $\geq 4$-fold was therefore very unlikely, which continues to question its value as gold standard for diagnosing $M p$ infection $[3,4]$. Its interpretation is also complicated by our observation of a $>4$-fold increase in $M p \operatorname{IgG}$ level in an asymptomatic sibling.

Besides describing $M p$ ASC response kinetics and duration, we also determined antibody specificity and isotype of the plasmablast response to infection, which are key to develop accurate ASC measurements [9]. We showed that $M p$-specific ASCs were not detectable in patients infected with influenza virus or EBV, whereas patients with influenza virus infection on the other hand developed influenza virus-specific ASC responses during CAP. The detection of $M p$ IgM ASCs was more sensitive than the detection of $M p$ IgG ASCs or $M p$ IgA ASCs for determining $M p$ infection, again in line with previous work [23].

The $M p$-specific ASC response was associated with clinical disease and correlated with CRP levels and WBC counts. Although $M p$ CAP was associated with lower CRP levels compared with CAP of other origin [14], severe disease defined as the presence of extrapulmonary skin manifestations was associated with increased systemic inflammation and higher CRP levels [21]. This finding suggests that in these children, inflammation may be indeed driven by $M p$ antigens, which warrants further investigation. It is interesting to note that there were no differences in ASC or antibody responses, or clinical outcomes such as fever duration, between groups with different antibiotic treatment regimens. In contrast, corticosteroid treatment significantly decreased ASC responses, although they were still detectable. One third of $M p$ IgM ASC-positive CAP patients were not treated with an antibiotic in vitro active against $M p$, but all of them equally and fully recovered. Although the study design does not allow conclusions about the effectiveness of treatment, these findings highlight the need for future interventional studies to assess the efficacy of antibiotics for $M p$ CAP $[2,31]$.

The ASC ELISpot assay is a robust technique $[9,32]$, and the protocol described here could be translated directly into the clinical setting to diagnose $M p$ infection by using only a small volume of peripheral blood $(\geq 1 \mathrm{~mL})$. However, the ASC ELISpot assay is labor-intensive, requiring the handling of fresh or frozen PBMCs [19, 32], and has a rather long overall turnaround time ( $\sim 24$ hours), but recent alternative protocols suggest more rapid ( $\sim 6-8$ hours) ASC detection [19]. Optimizing such protocols will help to routinely use the $M p$ IgM ASC ELISpot assay for diagnosing $M p$ CAP.

Despite variation in sampling time points, the study population represents a well defined cohort of children, both patients and controls, in whom pharyngeal swabs and fresh blood samples at several time points up to 6 months after inclusion could be obtained. It is challenging to collect blood samples from healthy children and already recovered children, and we can therefore not fully exclude any selection bias that occurred in the included subgroup of study participants. However, baseline characteristics of both included CAP patients and controls were similar compared with the enrolled study population. Some of the siblings of index patients were included as controls, which made it possible to increase the likelihood of detecting asymptomatic $M p$ carriers and to investigate the pattern symptom acquisition and/or changes in the results of the diagnostic tests. Mycoplasma pneumoniae was indeed more frequently detected in the URT of siblings compared with controls sampled during elective surgery ( $32 \%$ vs $4 \%$ ), which is in line with a higher $M p$ transmission rate within families and between other close contacts $[14,33,34]$. Proportionally, more siblings than other controls were included than were initially enrolled into the study ( $43 \%$ vs $12 \%)$, which may be partly due to a higher motivation of family members of affected patients agreeing to multiple sampling and follow-up. This may also explain the differences in $M p$ detection rates between included and enrolled study participants. A larger prospective confirmatory study is needed to validate these promising results of the Mp ASC ELISpot assay and to examine the added clinical utility of the ASC ELISpot assay to diagnose $M p$ infection in the clinical care of children with CAP.

\section{CONCLUSIONS}

In conclusion, $M p$-specific ASCs are short-lived and associated with clinical disease, in contrast to pharyngeal $M p$ DNA and serum antibodies. There are limited data on the duration of $\mathrm{B}$-cell responses during infection, and our study indicates that $M p$-specific peripheral blood B cells appear early in the course of illness, last only for several weeks before disappearing completely, and therefore they can be easily detected in a clinical setting with good diagnostic sensitivity and specificity. These findings expand our current knowledge on specific B-cell responses to infection, and they reveal ASCs as an optimal resource for determining disease etiology in $M p$ pneumonia and possibly other childhood infections.

\section{Supplementary Data}

Supplementary materials are available at The Journal of Infectious Diseases online. Consisting of data provided by the authors to benefit the reader, the posted materials are not copyedited and are the sole responsibility of the authors, so questions or comments should be addressed to the corresponding author.

\section{Notes}

Acknowledgments. We thank the following individuals: the children and their parents who contributed to this study; the emergency department staff, the division of anesthesiology staff, the division of otolaryngology staff, the outpatient clinic staff, and the short-stay department staff (University Children's Hospital Zurich) for recruiting participants; the microbiology laboratory staff (University Children's Hospital Zurich) for 
processing samples; and the primary care physicians and pediatricians for participating in out-of-hospital follow-up visits.

Author contributions. P. M. M. S., A. M. C. v. R., and C. B. contributed to study concept and design; P. M. M. S. contributed to acquisition of data; P. M. M. S., J. T., A. M. C. v. R., and C. B. contributed to data analysis and interpretation; P. M. M. S. and J. T. contributed to drafting the manuscript; all authors contributed to critical revision of the manuscript for important intellectual content; P. M. M. S. and J. T. contributed to statistical analysis; P. M. M. S., A. M. C. v. R., and C. B. obtained funding; P. M. M. S. and C. B. contributed to administrative, technical, or material support.

Disclaimer. The funders had no role in the study design, data collection and analysis, decision to publish, or preparation of the manuscript.

Financial support. P. M. M. S. was funded by a Fellowship Award from the European Society for Pediatric Infectious Diseases (ESPID) and grants from Promedica Foundation and Starr International Foundation.

Potential conflicts of interest. All authors: No reported conflicts of interest. All authors have submitted the ICMJE Form for Disclosure of Potential Conflicts of Interest.

\section{References}

1. Jain S, Williams DJ, Arnold SR, et al. Community-acquired pneumonia requiring hospitalization among U.S. children. N Engl J Med 2015; 372:835-45.

2. Gardiner SJ, Gavranich JB, Chang AB. Antibiotics for community-acquired lower respiratory tract infections secondary to Mycoplasma pneumoniae in children. Cochrane Database Syst Rev 2015; 1:CD004875.

3. Lee WJ, Huang EY, Tsai CM, et al. Role of serum Mycoplasma pneumoniae IgA, IgM, and IgG in the diagnosis of Mycoplasma pneumoniae-related pneumonia in schoolage children and adolescents. Clin Vaccine Immunol 2017; 24:e00471-16.

4. Waites KB, Xiao L, Liu Y, Balish MF, Atkinson TP. Mycoplasma pneumoniae from the respiratory tract and beyond. Clin Microbiol Rev 2017; 30:747-809.

5. Harris M, Clark J, Coote N, et al. British Thoracic Society guidelines for the management of community acquired pneumonia in children: update 2011. Thorax 2011; 66:ii1-23.

6. Bradley JS, Byington CL, Shah SS, et al.; Pediatric Infectious Diseases Society and the Infectious Diseases Society of America. The management of community-acquired pneumonia in infants and children older than 3 months of age: clinical practice guidelines by the Pediatric Infectious Diseases Society and the Infectious Diseases Society of America. Clin Infect Dis 2011; 53:e25-76.

7. Spuesens EB, Fraaij PL, Visser EG, et al. Carriage of Mycoplasma pneumoniae in the upper respiratory tract of symptomatic and asymptomatic children: an observational study. PLoS Med 2013; 10:e1001444.

8. Meyer Sauteur PM, Seiler M, Trück J, et al. Diagnosis of Mycoplasma pneumoniae pneumonia with measurement of specific antibody-secreting cells. Am J Respir Crit Care Med 2019; 200:1066-9.

9. Carter MJ, Mitchell RM, Meyer Sauteur PM, Kelly DF, Trück J. The antibody-secreting cell response to infection: kinetics and clinical applications. Front Immunol 2017; 8:630.

10. Ellebedy AH, Jackson KJ, Kissick HT, et al. Defining antigen-specific plasmablast and memory B cell subsets in human blood after viral infection or vaccination. Nat Immunol 2016; 17:1226-34.

11. Radbruch A, Muehlinghaus G, Luger EO, et al. Competence and competition: the challenge of becoming a long-lived plasma cell. Nat Rev Immunol 2006; 6:741-50.

12. Nutt SL, Hodgkin PD, Tarlinton DM, Corcoran LM. The generation of antibody-secreting plasma cells. Nat Rev Immunol 2015; 15:160-71.

13. Blanchard-Rohner G, Pulickal AS, Jol-van der Zijde CM, Snape MD, Pollard AJ. Appearance of peripheral blood plasma cells and memory B cells in a primary and secondary immune response in humans. Blood 2009; 114:4998-5002.

14. Meyer Sauteur PM, Krautter S, Ambroggio L, et al. Improved diagnostics help to identify clinical features and biomarkers that predict Mycoplasma pneumoniae community-acquired pneumonia in children. Clin Infect Dis 2019. doi:10.1093/ cid/ciz1059

15. British Thoracic Society of Standards of Care Committee. British Thoracic Society guidelines for the management of community-acquired pneumonia in childhood. Thorax 2002; 57:i1-24.

16. Cilla G, Oñate E, Perez-Yarza EG, Montes M, Vicente D, Perez-Trallero E. Viruses in community-acquired pneumonia in children aged less than 3 years old: high rate of viral coinfection. J Med Virol 2008; 80:1843-9.

17. Rhedin S, Lindstrand A, Hjelmgren A, et al. Respiratory viruses associated with community-acquired pneumonia in children: matched case-control study. Thorax 2015; 70:847-53.

18. Hardegger D, Nadal D, Bossart W, Altwegg M, Dutly F. Rapid detection of Mycoplasma pneumoniae in clinical samples by real-time PCR. J Microbiol Methods 2000; 41:45-51.

19. Saletti G, Çuburu N, Yang JS, Dey A, Czerkinsky C. Enzymelinked immunospot assays for direct ex vivo measurement of vaccine-induced human humoral immune responses in blood. Nat Protoc 2013; 8:1073-87.

20. R Core Team. R: A language and environment for statistical computing. R Foundation for Statistical Computing, Vienna, Austria. 2019. Available at: http://www.R-project. org/. Accessed 13 September 2019. 
21. Meyer Sauteur PM, Theiler M, Büttcher M, Seiler M, Weibel L, Berger C. Frequency and clinical presentation of mucocutaneous disease due to Mycoplasma pneumoniae infection in children with communityacquired pneumonia. JAMA Dermatol 2019. doi:10.1001/ jamadermatol.2019.3602

22. Azzi T, Lünemann A, Murer A, et al. Role for earlydifferentiated natural killer cells in infectious mononucleosis. Blood 2014; 124:2533-43.

23. Iseki M, Takahashi T, Kimura K, Yamashita R, Sasaki T. Number of specific antibody-secreting cells in the peripheral blood among children with mycoplasma pneumonia. Infect Immun 1996; 64:2799-803.

24. Fink K. Origin and function of circulating plasmablasts during acute viral infections. Front Immunol 2012; 3:78.

25. Palkola NV, Pakkanen SH, Kantele JM, Rossi N, Puohiniemi R, Kantele A. Pathogen-specific circulating plasmablasts in patients with pneumonia. PLoS One 2012; 7:e34334

26. Palkola NV, Blomgren K, Pakkanen SH, Puohiniemi R, Kantele JM, Kantele A. Immune defense in upper airways: a single-cell study of pathogen-specific plasmablasts and their migratory potentials in acute sinusitis and tonsillitis. PLoS One 2016; 11:e0154594.

27. Huang KY, Li CK, Clutterbuck E, et al. Virus-specific antibody secreting cell, memory B-cell, and sero-antibody responses in the human influenza challenge model. J Infect Dis 2014; 209:1354-61.
28. Habibi MS, Jozwik A, Makris S, et al.; Mechanisms of Severe Acute Influenza Consortium Investigators. Impaired antibody-mediated protection and defective IgA B-cell memory in experimental infection of adults with respiratory syncytial virus. Am J Respir Crit Care Med 2015; 191:1040-9.

29. Lee FE, Falsey AR, Halliley JL, Sanz I, Walsh EE. Circulating antibody-secreting cells during acute respiratory syncytial virus infection in adults. J Infect Dis 2010; 202:1659-66.

30. Lee FE, Halliley JL, Walsh EE, et al. Circulating human antibody-secreting cells during vaccinations and respiratory viral infections are characterized by high specificity and lack of bystander effect. J Immunol 2011; 186:5514-21.

31. Biondi E, McCulloh R, Alverson B, Klein A, Dixon A, Ralston S. Treatment of mycoplasma pneumonia: a systematic review. Pediatrics 2014; 133:1081-90.

32. Trück J, Mitchell R, Thompson AJ, et al. Effect of cryopreservation of peripheral blood mononuclear cells (PBMCs) on the variability of an antigen-specific memory B cell ELISpot. Hum Vaccin Immunother 2014; 10:2490-6.

33. Dorigo-Zetsma JW, Wilbrink B, van der Nat H, Bartelds AI, Heijnen ML, Dankert J. Results of molecular detection of Mycoplasma pneumoniae among patients with acute respiratory infection and in their household contacts reveals children as human reservoirs. J Infect Dis 2001; 183:675-8.

34. Walter ND, Grant GB, Bandy U, et al. Community outbreak of Mycoplasma pneumoniae infection: school-based cluster of neurologic disease associated with household transmission of respiratory illness. J Infect Dis 2008; 198:1365-74. 